\title{
Cadmium-induced Renopathy in Wistar Male Rats: Protection by Methanolic Leaf Extract of Momordica charantia Linn.
}

\author{
Ajilore, B.S. ${ }^{1}$, Ayannuga, O.A. ${ }^{2}$ and Oluogun, W.A. ${ }^{1}$ \\ ${ }^{I}$ (Department of Biochemistry, College of Health Sciences, Osun State University, Osogbo, Nigeria) \\ ${ }^{2}$ (Department of Anatomy, College of Health Sciences, Obafemi Awolowo University, Ile-ife, Nigeria) \\ Corresponding Author: Ajilore, B.S, Department of Biochemistry, College of Health Sciences, Osun State \\ University, Osogbo, Nigeria.
}

\begin{abstract}
The aim of present study was to evaluate the protective potentials of methanolic extract of the leaf of Momordica charantia (MC) against cadmium-induced renopathy in rats. Thirty male adult wistar rats were randomly divided into three groups $(A, B$ and $C)$ of ten rats each. Group A rats served as the control and received normal saline orally. Group B rats were treated with cadmium chloride $2.5 \mathrm{mg} / \mathrm{kg}$ bwt subcutaneously while group C rats were pre-treated orally with extract of $M C 300 \mathrm{mg} / \mathrm{kg}$ bwt before treating with cadmium chloride $2.5 \mathrm{mg} / \mathrm{kg}$ bwt subcutaneously. The rats were treated every other day regularly for six weeks. Blood samples were collected by ocular puncture and five rats each per group were sacrificed at third week and six week post-treatment intervals. Serum creatinine and urea were evaluated. Histopathology of the kidney was also studied. In cadmium only-treated rats, serum creatinine and urea levels were significantly $(p<0.05)$ increased when compared to control rats at the two time intervals. Histological observations showed varying degree of renal toxicity. However, the toxic effect of cadmium was significantly controlled in the rats pre-treated with methanolic extract of $M C$ at the two time intervals.
\end{abstract}

Keywords: Momordica charantia, cadmium chloride, renopathy, protective potentials.

\section{Introduction}

Cadmium is well-known heavy metal present in the environment and causes serious environmental and occupational hazards to humans [1, 2]. Both acute and chronic exposure to cadmium can damage various organs including kidney, liver, testes, lungs, and bone depending on the dose, route and duration of the exposure. In humans, the kidney is recognized as the most critical organ affected by chronic exposure to cadmium [3, 4], whereas acute exposure to cadmium mainly affects the liver [5]. Kidney damage is the initial site of damage in cases of low-level chronic inhalation exposure or oral exposure, and the lung is the initial site of damage in high-level inhalational exposure [6]. During the chronic exposure the metal may interfere with metabolic process via renal cortex resulting in renal dysfunction [2]. Chronic cadmium intoxication causes focal cortical damage in humans and apoptosis of the proximal tubular cells has been demonstrated in rats and dogs [7].

Momordica charantia is a tendril bearing economically important medicinally vine belonging to the family cucurbitaceae $[8,9]$. It is a vegetable widely cultivated in tropical areas including West Africa, East Africa, Amazon, and the Carribean, both as medicine and fruit. All the plant parts including flower, leaves, stem, fruits and seeds have been used traditionally to treat array of conditions like diabetes, hypertension, cancers, ulcers, asthma, Gastrointestinal problems, fever, inflammation, erectile dysfunction, bacteria and viral infections [10]. This study was designed to investigate renoprotection of the methanolic leaf extract of Momordica charantia on the serum urea and creatinine, and histology of cadmium-induced kidney damage.

\subsection{Plant Material}

\section{Materials And Methods}

Fresh leaves of Momordica charantia (Bitter melon) were collected form a farm in Osu, Osun State, Nigeria. The plant was identified and authenticated at the Ife Herbarium, Department of Botany, Obafemi Awolowo University, Nigeria, where the specimen copy was deposited. The Herbarium identification number was 16591.

\subsubsection{Preparation of Methanolic extract of Plant material}

The leaves of Momordica charantia were shade dry under laboratory conditions for thirty (30) days and ground into fine powder by using an electrical mill. The powder was kept in air-tight container until use. Three hundred and fifty gram $(350 \mathrm{~g})$ of the dried powder was subjected to soxhlet extraction with 3.5 litres of $70 \%$ (v/v) methanol for two (2) consecutive days using modified method of Virdii et al., 2003 [11]. $500 \mathrm{mls}$ of warm water was added to the mixture to suspend the chlorophyll. The mixture was filtered to remove the suspended chlorophyll and filtrate was evaporated to dryness under reduced pressure using Buchi Rotary 
Evaporator and the yield was calculated. The extraction was done at DRPU (Drug Research and Production Unit) in the Faculty of Pharmacy, Obafemi Awolowo University, Ile-ife, Nigeria.

\subsection{Experimental Animals}

Thirty (30) adult male wistar rats weighing $200 \mathrm{~g}$ to $250 \mathrm{~g}$ were used for this study. The animals were acclimatized for 2 weeks at the Animal House of the College of Health Sciences, Obafemi Awolowo University, Nigeria where they had free access to standard pellets (Mosodun Feeds, Osogbo, Nigeria) and clean water.

\subsection{Experimental Design}

Thirty (30) adult male wistar rats weighing between $200 \mathrm{~g}$ and $250 \mathrm{~g}$ were randomly divided into 3 groups, A, B and C of 10 rats each. A was the control group and received Normal saline, B was cadmium only treated group and group $\mathrm{C}$ was pre-treated with methanolic extract of Momordica charantia one hour before treating with cadmium. The rats were treated every other day regularly for six weeks as follows:

Group A (Normal saline) - Each rat in this group received $1 \mathrm{ml}$ per day $0.9 \%$ w/v of Normal saline. Route of administration was per oral.

Group B (Cadmium only) - Each rat in this group received $2.5 \mathrm{mg} / \mathrm{kg}$ body weight Cadmium per day. Route of administration was subcutaneous.

Group C (Extract + Cadmium) - Each rat in this group was pre-treated with $300 \mathrm{mg} / \mathrm{kg}$ body weight methanolic extract of Momordica charantia per oral one hour before subcutaneous $2.5 \mathrm{mg} / \mathrm{kg}$ body weight Cadmium was given.

All the rats in the three groups were treated every other day regularly for six weeks. Five blood samples were collected by ocular puncture after 3 and 6 weeks of treatment and sera separated for various biochemical assays. Five rats were sacrificed by cervical decapitation at the two time intervals previously mentioned and kidney tissues were removed, cleaned, and immediately fixed in $10 \%$ formalin for histological study.

\subsection{Biochemical Renal Function Estimations}

The renal function parameters estimated from the serum were urea and creatinine. The urea and creatinine levels in the serum were determined by Randox diagnostic kits (Randox Laboratory Ltd., Crumlin, County Antrim, UK) by method of Faweett and Scott,1960 [12].

\subsection{Histopathological Studies}

Five rats were sacrificed by cervical decapitation at the two time intervals previously mentioned and kidney tissues were removed, cleaned, and immediately fixed in $10 \%$ formalin. The tissues were transferred into an automatic processor where they went through a process of dehydration. The tissues were then cleared in Xylene and embedded in paraffin wax. Serial sections of 5 micron thick were obtained using a rotary microtome. The tissue sections were deparaffinised hydrated and stained using the routine haematoxylin and eosin staining method (H\&E). The stained sections were examined under the light microscope.

\subsection{Statistical Analysis}

The data were expressed as the mean \pm SE. Statistical difference between groups were assessed by paired-samples T-test using SPSS package (version 16.0) and $\mathrm{p}$ values $<0.05$ were considered significant.

\subsection{Serum creatinine and urea concentrations}

\section{Results}

Table 1 showed the effect of cadmium and extract of Momordica charantia (MC) on serum urea and creatinine levels at three and sixth week post-treatments. In cadmium only-treated rats serum creatinine levels were significantly $(\mathrm{p}<0.05)$ increased when compared to control rats at the two time treatment intervals. However, serum creatinine levels in rats pre-treated with extract of MC were significantly $(p<0.05)$ reduced when compared with cadmium only-treated rats. There was no significant $(\mathrm{p}<0.05)$ difference in serum creatinine levels of control rats and those pre-treated with extract of MC. Serum urea level of cadmium onlytreated rats was increased at three week post-treatment (though not significant at $\mathrm{p}<0.05$ ). However, at sixth week post-treatment, there was significant $(p<0.05)$ increase in the level of serum urea of rats treated-only with cadmium when compared to control rats and those pre-treated with extract of Momordica charantia.

\subsection{Histological studies}

Figures 1 and 2 showed the histological studies of the renal tissues of the control, cadmium-only treated, and MC extracts pre-treated rats at the third week and sixth week post-treatment stages respectively. The histological analysis of the renal cortex revealed that control rats showed normal morphology. In cadmiumonly treated rats, there was significant reduction of the glomerular spaces at the two-time treatment intervals. 
There was recovery of glomerular spaces in rats pre-treated with methanolic extract of Momordica charantia (MC). There was also slight clumping of nucleus in rats pre-treated with extract of $\mathrm{MC}$ at the end of three weeks treatment. There was significant deposit of blue-stained materials (thought to be cadmium deposits) which further obscured the outline of renal corpuscles in cadmium-only treated rats. There was slight deposit of bluestained materials in rats pre-treated with extract of MC. Macula densa was not obvious in all rats challenged with cadmium.

\section{Discussion And Conclusion}

Cadmium is also one of the most toxic industrial and environmental metals that cause health hazard [13]. Nephrotoxicity caused by cadmium has been described in settings of industrial exposure and environmental pollution [14]. The current study was focused at determining the protective potential of methanolic extract of the leaf of Momordica charantia (MC) on cadmium-induced renal damage in rats. The result of our study supported the fact that cadmium induces renal toxicity $[7,15,2,1]$. The results of this study also showed that cadmium-induced renal damage increases serum creatinine and urea levels. This is in agreement with previous studies $(16,17,18,19,20]$. Pathogenesis of cadmium-induced renal damage has been attributed to the binding of cadmium to a low molecular weight binding protein called methalothionein. More than $80 \%$ of cadmium is bound to methalothionein. Methalothionein is a protective protein found largely in liver and kidney [16]. Urea and creatinine levels are used to monitor renal function and their levels will not rise until at least half of the kidney nephrons are destroyed [19]. The results of our study also showed that methanolic leaf extract of Momordica charantia (MC) improved renal function of the rats pre-treated with the extract. This activity may be due to pronounced antioxidant properties of the plant of study by reducing oxidative damage to the microstructure of the kidney [21].

Cadmium-induced kidney damage was also evaluated using histological approach. The results of our study further showed that cadmium causes renal damage. There was significant reduction of the glomerular spaces which was responsible for significant increase in the biochemical parameters such as creatinine and urea. There was also significant cadmium deposits in the cortex of the rats treated with cadmium alone. Deposition of cadmium in the cortex may be due to binding of cadmium to small molecular weight protein, metallothionein $[22,1]$. However, cadmium-induced renal damage was restored to near normal morphology by methanolic leaf extract of Momordica charantia (MC). The ability of extract of MC to prevent oxidative damage caused by heavy metals like cadmium may be due to its anti-lipid peroxidation activity and antioxidant property [21, 23].

In conclusion, we therefore inferred that cadmium induces toxic renal damage, and methanolic leaf extract of Momordica charantia has appreciable potentials to prevent damage to the kidney.

\section{Acknowledgement}

Authors acknowledge the support provided by the Department of Biochemistry, College of Health Sciences, Osun State University, Osogbo and the Department of Anatomy, College of Health Sciences, Obafemi Awolowo University, Ile-Ife. We are also thankful to the staffs of Drug Research and Production Unit of the Faculty of Pharmacy, Obafemi Awolowo University, Ile-Ife. Finally, we acknowledge the help received from the scholars whose articles are cited and included in references of the manuscript.

\section{References}

[1] Tarasub, N., Tarasub, C. and Ayuthaya, W.D. (2011). Protective role of curcumin on cadmium induced nephrotoxicity in rats. $J$. Environ. Chem. Ecotoxicol. 3(2): 17-24.

[2] Siddiqui, M.F. (2010). Cadmium induced renal toxicity in male rats, Rattus rattus. East. J. of Med. 15: 93-96

[3] Foulkes, E.C. and Blanck, S. (1990). Acute cadmium uptake by rabbit kidneys: Mechanism and effects. Toxicol Appl Pharmacol. 102: 464-473.

[4] Trian, E.K. and Trian, A. (1995). Age dependency of selenium and cadmium content in human liver, kidney and thyroid. Arch. Environ. Health. 50: 242-246.

[5] Habbeebu, S.S., Liu, J. and Klaassen, C.D. (1998). Cadmium-induced apoptosis in mouse liver. Toxicol Appl Pharmacol 149(2):203-209.

[6] Kido, T., Nogawa, K., Ishizaki, M., Honda, R., Tsuritani, I. and Yamada, Y. (1990). Long-term observation of serum creatinine and arterial blood $\mathrm{pH}$ in persons with cadmium-induced renal dysfunction. Arch. Environ. Health. 5: 35-41.

[7] Aoyagi, T., Hayakawa, K., Miyaji, K., Ishikawa, H. and Hata, M. (2003). Cadmium nephrotoxicity and evacuation from the body in a rat modeled subchronic intoxication. Int. J. Urol. 10: 332-338.

[8] Paul, A. and Raychaudhuri, S.S. (2010). Medicinal uses and molecular identification of two Momordica charantia varieties- A review. Electronic Journal of Biology. 6(2): 43-51.

[9] Bakare, R.I., Magbagbeola, O. A., Akinwande, A. I. and Okunowo O. W. (2010). Nutritional and chemical evaluation of Momordica charantia. Journal of Medicnal Plant Research. 4(21): 2189-2193.

[10] Kumar, K.P.S. and Bhowmik, D. (2010). Traditional medicinal uses and therapeutic benefits of Momordica charantia Linn. Int J Pharm sci Review \& Research 4(3): 23-28.

[11] Virdi, J., Sivakami, S., Shahani, S., Suthar, A.C., Banavalikar, M.M. and Biyani, M.K. (2003). Antihyperglycemic effect of three extracts from Momordica charantia. J Ethnopharmacol. 88(1): 107-111.

[12] Faweett, J. K. and Scott, J.E. (1960). Clin. Path. 13: 156. 
[13] Alhazza, I.M. (2008). Cadmium-induced hepatotoxicity and oxidative stress in rats: Protection by selenium. Research Journal of Environmental Sciences. 2(4): 305-309.

[14] Gonick, H.C. (2008). Nephrotoxicity of cadmium and lead. Indian J. Med. Res. 128: 335-352.

[15] Chen, L., Jin. T., Huang, B., Nordberg, G. and Nordberg, M. (2006). Critical exposure level of

[16] cadmium for elevated urinary metallothionein-An occupational population study in China. Toxicol. Appl. Pharmacol. 215 : $93-9$.

[17] Moshtaghie, A.A., Raisi, A. and Goodarzi, H. (1991). A study of the effect of cadmium toxicity on serum proteins and its relation to proteinuria in male rats. J. of Islam. Acad. of Sci. 4 (3): 192-195.

[18] Kowalczyk, E., Jankowski, A., Niedworok, J., Śmigielski, J. and Tyslerowicz, P. (2002). Effect of long-term cadmium intoxication on selected biochemical parameters in experimental animals. Polish J. of Environ. Studies. 11(5): 599-601.

[19] Gaurav, D., Preet, S. and Dua, K.K. (2010). Protective effect of Spirulina platensis on cadmium induced renal toxicity in wistar rats. Archives of Appl. Sci. Res. 2(1): 390-397.

[20] Gaurav, D., Preet, S. and Dua, K.K. (2011). Protective influence of dietary nutrients on antioxidant defense system in the blood of rats treated with cadmium. Advances in Appl. Sci. Res. 2(2): 69-78

[21] Obianime, A. W., Aprioku, J. S. and Ahiwe, N. J. (2011). Biochemical and hormonal effects of cadmium in female guinea pigs. J. Toxicol. Environ. Health Sci. 3(2): 39-43.

[22] Teoh, S.L., Abd Lattif, A. and Das, S. (2010). Histological changes in the kidneys of experimental diabetic rats fed with Momordica charantia (bitter gourd) extract. Rom. J. of Morpho. and Embryo. 51(1): 91-95.

[23] Okubo, A., Hanafusa, M., Imada, M., Yamashita, A., Yamazaki, S. and Toda, S. (1991). Accumulation of cadmium and morphological changes of kidney observed by scanning electron microscope. Analyt. Sci., 7: 815-816.

[24] Abdollahi, M., Zuki, A.B.Z., Rezaeizadeh, A., Goh, Y.M. and Noordin, M.M. (2011). Effects of Momordica charantia aqueous extract on renal histopathological changes associated with streptozotocin-induced diabetes mellitus type II in neonatal rats. J. Med. Plant. Res. 5(9): 1779-1787.

Table 1: Effect of Cadmium and Extract of Momordica charantia (MC) on Serum Urea (mmol/L) and Creatinine, CREA, $(\mu \mathrm{mol} / \mathrm{L})$ of Male Rats.

\begin{tabular}{|c|c|c|c|c|c|c|}
\hline \multicolumn{7}{|c|}{ Treatment Group } \\
\hline \multicolumn{3}{|c|}{ Control } & \multicolumn{2}{|l|}{ Cadmium } & \multicolumn{2}{|c|}{ Cadmium and Extract of MC } \\
\hline Weeks & UREA & CREA & UREA & CREA & UREA & CREA \\
\hline$\overline{3}$ & $14.13 \pm 0.64$ & $71.99 \pm 7.63$ & $16.31 \pm 1.01$ & $104.35 \pm 13.12 * *$ & $14.36 \pm 1.04$ & $76.04 \pm 6.54^{\prime \prime}$ \\
\hline 6 & $8.61 \pm 0.01$ & $68.74 \pm 0.02$ & $10.81 \pm 0.77 *$ & $* * 101.54 \pm 11.16^{* *}$ & $8.09 \pm 2.06$ & $79.01 \pm 8.92^{*}$ \\
\hline
\end{tabular}

Values are expressed as mean \pm SEM $(n=5)$. **Significantly different from control, $\mathrm{p}<0.05$; "Significantly different from cadmium group, $\mathrm{p}<0.05$.
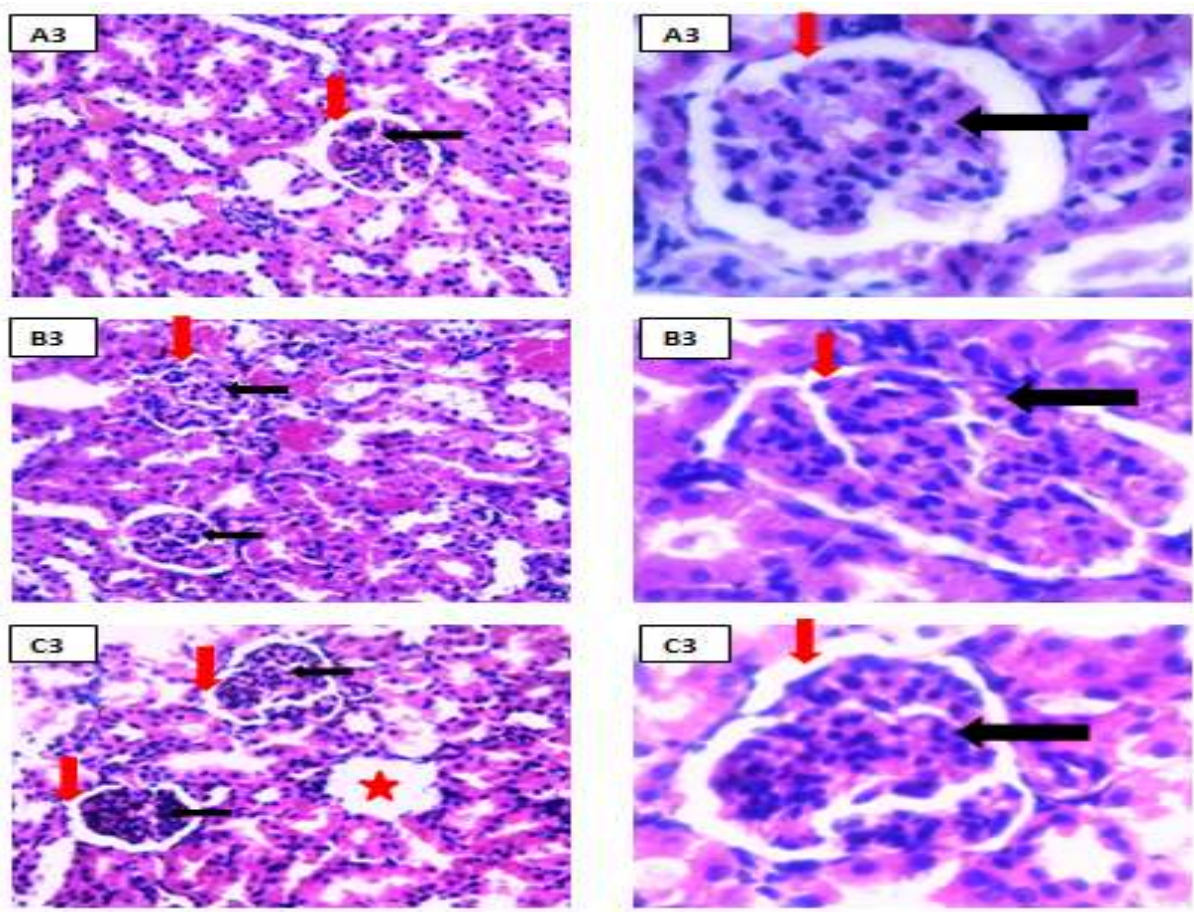

Figure 1: Photomicrographs of the renal cortex showing the renal corpuscle (black arrow). There is slight clumping of the glomerulus in $\mathrm{C}$ slides as well as an empty renal corpuscle. Glomerular space (red arrow) is markedly removed in B slides while there is recovery of the glomerular space in C slides. 
Stain H\&E. Mag. X400 Left Panel, X1000 Right Panel.
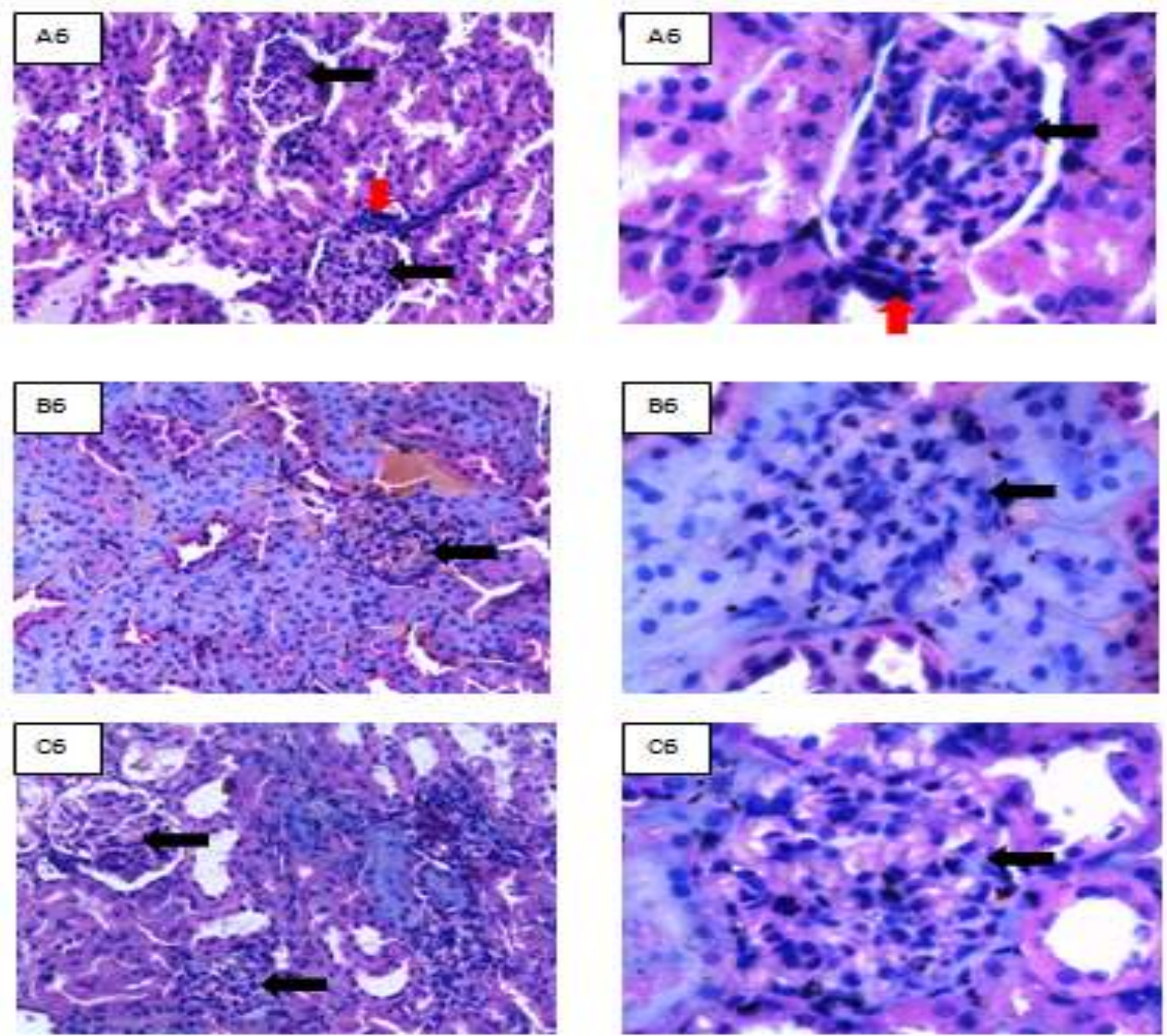

Figure 2: Photomicrographs of the renal cortex of A (control), B (cadmium only) and C (MC extract pretreated) rats at 6th week post-treatment stage showing the renal glomeruli (black arrow), macula densa (red arrow). The cellular components of the renal corpuscle of B are closely packed with obliteration of the glomerular space. A bluish stained deposit further obscures the outline of the corpuscle in the B slides. A lesser obliteration of the glomerular space is seen in the $\mathrm{C}$ slides, with lesser deposit of the bluish stained material. Macula densa is not obvious in the $\mathrm{B}$ and $\mathrm{C}$ renal corpuscle.

Stain H\&E. Mag. X1000 Right panel, x400 Left panel. 